\title{
Punicalagin ameliorates spermatological parameters in bisphenol A treated New Zealand White Rabbits
}

\author{
OZLEM YILDIZ-GULAY, GULSAH CANDAN*, MEHMET SUKRU GULAY
}

\begin{abstract}
Burdur Mehmet Akif Ersoy University, Faculty of Veterinary Medicine, Department of Physiology Burdur, Turkey
\end{abstract} ${ }^{*}$ Bucak Public Hospital, Burdur, Turkey

Yildiz-Gulay O., Candan G., Gulay M. S.

\section{Punicalagin ameliorates spermatological parameters in bisphenol A treated New Zealand White Rabbits}

\section{Summary}

The ameliorating effects of punicalagin (PUN) were evaluated against some reproductive parameters in male New Zealand White rabbits treated with bisphenol A $(B P A)$. Rabbits $(n=24)$ were adapted to laboratory conditions for 2 weeks before the experiment. After adaptation, rabbits were randomly assigned into 4 groups. The control group received tap water and corn oil. Rabbits in BPA and PUN groups received $20 \mathrm{mg} / \mathrm{kg} \mathrm{BPA}$ in corn oil + tap water, and $2 \mathrm{mg} / \mathrm{kg}$ PUN in tap water + corn oil, respectively. Rabbits in the last group received the same amounts of BPA and PUN. All treatments were administered by daily oral gavages for 9 weeks. On d 63 of the experiment, semen and blood samples were collected. Blood samples were used for the evaluation of some hormones. After blood collection, rabbits were euthanized and the right testis was stored at $-80^{\circ} \mathrm{C}$. At the end of the experiment, inhibin, FSH, $\mathrm{LH}, \mathrm{FSH} / \mathrm{LH}$ ratio, FSH/inhibin ratio, and estrogen levels were similar among the groups. However, testosterone levels in serum, and superoxide dismutase, catalase, and malondialdehyde levels in testis tissues were negatively affected by BPA. Moreover, sperm concentrations and the percent of progressive motility significantly declined in BPA treated rabbits. PUN treatment significantly improved these parameters up to control levels when coupled with BPA. The results indicated that PUN has ameliorating effects in neutralizing the possible toxic effects of BPA in male rabbits.

Keywords: rabbit, semen, reproductive hormones, punicalagin, bisphenol A, oxidative degeneration

Industry's incredible progress in recent decades has increased the risk of environmental contamination by endocrine-disrupting chemicals (EDC) (28). Nowadays, one of the highest produced EDC is bisphenol A (BPA). As a result, BPA is very common in our environment, and all animals can be exposed to BPA from both nutritional and non-nutritional sources (9). Detection of BPA in human serum, urine, placental tissue, umbilical cord blood, and breast milk is evidence of how widespread is environmental pollution because of this chemical $(9,24)$. Due to the increase in BPA exposure rates and the increasing concern about possible health effects, regulations regarding the production and use of BPA have been introduced in recent years (6).

Punicalagin is one of the largest known polyphenols commonly found in pomegranate fruit. It has a molar mass of $1084.722 \mathrm{~g} / \mathrm{mol}$. Punicalagin has strong antioxidant features since it has a total of 16 degradable $-\mathrm{OH}$ group. Punicalagin is part of the family of the ellagitannins. The ellagitannins are a diverse class of hydrolyzable tannins such as punicalin and gallic acid. As a polyphenol, punicalagin contains gallic acid and glucose-bound ellagic acid. Thus all these ellagitan- nins, including punicalagin, are capable of hydrolyzing to ellagic acid (10). In this way, they cause the ellagic acid to be released into the blood for a long time.

There are strong pieces of evidence that BPA can negatively alter testosterone production, sperm count, sperm motility, and overall spermatogenesis and sperm quality in a man and different mammals $(11,18,35)$. Moreover, the results from our laboratory suggested as low as $10 \mathrm{mg} / \mathrm{kg} / \mathrm{d}$ dose of oral BPA had negative effects on serum testosterone, progressive motility, and spermatozoa concentration in New Zealand White rabbits by changing the oxidant-antioxidant status of the testicular tissues (16). Recently, it has been reported that, due to its high antioxidant capacity, $2 \mathrm{mg} / \mathrm{kg} / \mathrm{day}$ punicalagin had a positive effect on antioxidant activity in testicular tissues and increased sperm count in New Zealand rabbits (34). Thus, these positive effects of punicalagin suggested that it can be used to reduce or prevent the possible toxic effects of BPA on testicular tissue and spermatogenesis.

Therefore, the current study aimed to investigate the possible protective effects of this natural antioxidant in male New Zealand rabbits against oral BPA. 


\section{Material and methods}

Experimental design. The trial was carried out at the Experimental Animals Unit of Burdur Mehmet Akif Ersoy University, Faculty of Veterinary Medicine. The study was approved by the Ethics Committee of the Burdur Mehmet Akif Ersoy University (25.11.2015/159) and was supported partly by TUBITAK (116O027) and Burdur Mehmet Akif Ersoy University Scientific Research Projects Unit (BAP0474YL-17). A total of 24 male New Zealand White rabbits were individually housed in galvanized cages. The ages and weights of the rabbits were $8-10$ months and $2.8-3.7 \mathrm{~kg}$, respectively. The bucks were trained to use an artificial vagina for 14 days before the experiment and kept at standard laboratory conditions (50-55\% humidity, $22 \pm 2{ }^{\circ} \mathrm{C}$ temperature, and $14: 10$ hours of light: dark cycle) during the entire experimental period.

Water and feed were given as ad libitum. The rabbits were fed with standard commercial rabbit feed (Korkuteli Food Company, Antalya, Turkey; $6.93 \%$ crude ash, $17.0 \%$ crude protein, $12.68 \%$ crude cellulose, $3.67 \%$ crude oil, $0.49 \%$ calcium, $0.46 \%$ phosphorous). Body weights of the rabbits were taken weekly and dose adjustments of BPA were made according to their body weights.

After the training period, the rabbits randomly divided into 4 groups of 6 rabbits per treatment group. The study protocol for the groups used during the current study was as follows:

Control group (C): Daily oral corn oil (1 mL corn oil for $1 \mathrm{~kg}$ live weight $)+$ daily oral distilled water $(1 \mathrm{~mL}$ distilled water for $1 \mathrm{~kg}$ live weight);

Bisphenol A group (BPA): Daily oral BPA (20 mg/kg live weight) in corn oil (1 mL corn oil contained $20 \mathrm{mg}$ BPA) and daily distilled water (1 $\mathrm{mL}$ distilled water for $1 \mathrm{~kg}$ live weight);

Punicalagin group (PUN): Daily oral punicalagin $(2 \mathrm{mg} /$ $\mathrm{kg}$ live weight $)$ in distilled water $(1 \mathrm{~mL}$ distilled water contained $2 \mathrm{mg}$ punicalagin) and daily oral corn oil ( $1 \mathrm{~mL}$ corn oil for $1 \mathrm{~kg}$ live weight);

Bisphenol A+Punicalagin group (B+P): Daily oral BPA $(20 \mathrm{mg} / \mathrm{kg}$ live weight $)$ in corn oil $(1 \mathrm{~mL}$ corn oil contained $20 \mathrm{mg}$ BPA $)$ and daily oral punicalagin $(2 \mathrm{mg} / \mathrm{kg}$ live weight $)$ in distilled water $(1 \mathrm{~mL}$ distilled water contained $2 \mathrm{mg}$ punicalagin).

The appropriate doses were chosen for BPA (16) and punicalagin (34) in the lights of previous experiments. Oral gavage practices were performed every day between 08:00 and 09:30 hours before the morning feedings.

Blood collection. At the end of oral gavage practices (63 days), rabbits were fasted for 12 hours and blood samples were collected from the ear artery with the help of $24 \mathrm{G}$ intravenous cannula. Blood samples were centrifuged at $1457 \times \mathrm{g}$ for $20 \mathrm{~min}$ and serum samples were stored at $-20^{\circ} \mathrm{C}$ until analysis.

Semen collection and evaluation procedures. The semen collections were performed according to Ata et al. (4). Immediately after the collection, the semen samples were examined for spermatological parameters $(3,4)$. Spermatological parameters such as ejaculate volume, ejaculate weight, semen $\mathrm{pH}$, spermatozoa concentration, and sperm motility were calculated from the ejaculate samples taken at the end of the $9^{\text {th }}$ week to see the possible effects of BPA and punicalagin treatments as explained elsewhere $(3,4,16)$.

Reproductive hormone analysis. Serum levels of some reproductive hormones were determined using rabbit specific ELISA kits. Serum samples of each rabbit were analyzed for rabbit testosterone (Cat No CSB-E06927Rb, Cusabio Biotech Co., LTD.; intra- and inter assay precision of $<15 \%$ and the detection range of $0.625-10 \mathrm{ng} / \mathrm{mL}$ ), rabbit estradiol (Cat No CSB-E069915Rb, Cusabio Biotech Co., LTD.; intra- and inter assay precision of $<15 \%$ and the detection range of $62.5-1000 \mathrm{pg} / \mathrm{mL})$, rabbit luteinizing hormone - LH (Cat No: SG-0004Rb, SinoGeneClon Biotech Co., Ltd.; intra- and inter assay precision of $<10 \%$ and the detection range of 3-100 $\mathrm{mIU} / \mathrm{mL}$ ), rabbit follicle-stimulating hormone - FSH (Cat No: SG-0001Rb; SinoGeneClon Biotech Co., Ltd.; intra- and inter assay precision of $<10 \%$ and the detection range of $5-180 \mathrm{mIU} / \mathrm{mL}$ ) and rabbit inhibin alpha - INHA (Cat No: SG-0015Rb; SinoGeneClon Biotech Co., Ltd.; intra- and inter assay precision of $<15 \%$ and the detection range of 33-2000 $\mathrm{pg} / \mathrm{mL}$ ). Measurements were performed according to the manufacturer's instructions.

Oxidant-antioxidant parameters. The right testis from each rabbit was collected immediately after euthanasia and were washed with PBS chilled to $5^{\circ} \mathrm{C}$ and stored at $-80^{\circ} \mathrm{C}$ until used for oxidant and antioxidant parameters. Before the analysis, the testis tissues were prepared according to the manufacturer's instructions. Superoxide dismutase SOD (Cat No: SG-0061Rb; intra- and inter assay precision of $<10 \%$ and the detection range of $30-1000 \mathrm{pg} / \mathrm{mL}$ ), glutathione peroxidase - GPx (Cat No: SG-0120Rb, intraand inter assay precision of $<10 \%$ and the detection range of 33-3000 pg/mL), catalase - CAT (Cat No: SG-50185; intra- and inter assay precision of $<10 \%$ and the detection range of 1-36 pg/mL), and malondialdehyde - MDA (Cat No: SG-50252; SinoGeneClon Biotech Co., Ltd.; intra- and inter assay precision of $<10 \%$ and the detection range of 0.3-7 mmol/L) levels in the testis tissues were assayed using ELISA kits (SinoGeneClon Biotech Co., Ltd., China). The results were read at $450 \mathrm{~nm}$.

Histopathological examination. At necropsy, the testis tissues were fixed in 10\% formaldehyde. The tissues passed through the routine procedure and were blocked in paraffin. The paraffin embedded blocks were cut by microtome at a thickness of $5 \mu$. The samples were stained with hematoxylin-eosin and examined under the criteria by Jahan et al. (13). Degeneration of Sertoli cells, shedding of germ cells, and the amount of sperm in the tubule lumen were evaluated.

Statistical analyses. All values are given as mean $\pm \mathrm{SD}$. PROC ANOVA procedure of the SAS statistical program was used for statistical evaluation. The individual means of treatments were compared with the Tukey test. In all statistical applications the difference was considered significant when the difference among the groups was $\mathrm{P}<0.05$.

\section{Results and discussion}

The data for reproductive hormones and spermatological parameters are in Table 1 and 2, respectively. Among the reproductive hormones studied, only the serum levels of testosterone were affected by BPA exposure and serum testosterone levels declined in BPA 
Tab. 1. Effects of oral bisphenol A (BPA) and/or punicalagin (PUN) administration on some reproductive hormones in male New Zealand White Rabbits $(n=6)$

\begin{tabular}{|l|c|c|c|c|c|}
\hline & CONT & BPA & PUN & B+P & P \\
\hline Testosterone $(\mathrm{ng} / \mathrm{mL})$ & $4.18^{\mathrm{a}} \pm 0.50$ & $3.30^{\mathrm{b}} \pm 0.34$ & $4.00^{\mathrm{a}} \pm 0.50$ & $3.59^{\mathrm{ab}} \pm 0.30$ & 0.023 \\
Estrogen $(\mathrm{pg} / \mathrm{mL})$ & $472.4 \pm 81.4$ & $692.8 \pm 284$ & $653.2 \pm 220$ & $595.4 \pm 148.8$ & 0.356 \\
Inhibin $(\mathrm{pg} / \mathrm{mL})$ & $165.5 \pm 16.3$ & $157.3 \pm 13.1$ & $169.4 \pm 10.5$ & $167.7 \pm 11.9$ & 0.164 \\
\hline FSH $(\mathrm{mIU} / \mathrm{mL})$ & $9.37 \pm 1.76$ & $8.88 \pm 1.40$ & $10.04 \pm 1.21$ & $10.26 \pm 1.67$ & 0.233 \\
LH $(\mathrm{mlU} / \mathrm{mL})$ & $9.62 \pm 1.17$ & $9.59 \pm 1.08$ & $9.66 \pm 1.15$ & $10.27 \pm 1.37$ & 0.392 \\
FSH/lnhibin ratio $(\%)$ & $0.056 \pm 0.003$ & $0.056 \pm 0.003$ & $0.059 \pm 0.003$ & $0.060 \pm 0.006$ & 0.320 \\
\hline FSH/LH ratio (\%) & $0.97 \pm 0.16$ & $0.92 \pm 0.14$ & $1.04 \pm 0.13$ & $1.00 \pm 0.17$ & 0.443 \\
\hline
\end{tabular}

Explanations: CONT - control; BPA - $20 \mathrm{mg} / \mathrm{kg} / \mathrm{day}$ BPA; PUN - $2 \mathrm{mg} / \mathrm{kg} /$ day punicalagin; $\mathrm{B}+\mathrm{P}-20 \mathrm{mg} / \mathrm{kg} /$ day BPA and $2 \mathrm{mg} / \mathrm{kg} /$ day punicalagin; $\mathrm{a}, \mathrm{b}$ - rows with different letters differ significantly. Values are given as mean \pm standard deviation.

Tab. 2. Effects of oral bisphenol A (BPA) and/or punicalagin (PUN) administration on some spermatological parameters in male New Zealand White Rabbits $(n=6)$

\begin{tabular}{|l|r|r|r|r|c|}
\hline & \multicolumn{1}{|c|}{ CONT } & \multicolumn{1}{c|}{ BPA } & \multicolumn{1}{c|}{ PUN } & \multicolumn{1}{c|}{ B+P } & P \\
\hline Volume $(\mathrm{mL})$ & $0.83 \pm 0.20$ & $0.80 \pm 0.09$ & $0.85 \pm 0.10$ & $0.81 \pm 0.09$ & 0.908 \\
Weight $(\mathrm{mg})$ & $0.91 \pm 0.20$ & $0.80 \pm 0.21$ & $0.90 \pm 0.13$ & $0.84 \pm 0.08$ & 0.614 \\
Progressive Motility $(\%)$ & $70.8^{\mathrm{b}} \pm 3.76$ & $45.8^{\mathrm{c}} \pm 8.61$ & $80.8^{\mathrm{a}} \pm 5.84$ & $65.0^{\mathrm{b}} \pm 2.74$ & 0.001 \\
pH & $7.13 \pm 0.12$ & $7.11 \pm 0.14$ & $7.10 \pm 0.06$ & $7.11 \pm 0.09$ & 0.965 \\
Concentration $\left(\times 10^{6} / \mathrm{mL}\right)$ & $285.1^{\mathrm{b}} \pm 67.3$ & $207.5^{\mathrm{c}} \pm 33.2$ & $351.1^{\mathrm{a}} \pm 37.1$ & $264.0^{\mathrm{b}} \pm 36.6$ & 0.001 \\
SPP $(\mathrm{g} / \mathrm{dL})$ & $3.51 \pm 0.49$ & $3.38 \pm 0.31$ & $3.41 \pm 0.27$ & $3.50 \pm 0.49$ & 0.928 \\
\hline
\end{tabular}

Explanations: as in Tab. 1.

Tab. 3. Effects of oral bisphenol A (BPA) and/or punicalagin (PUN) administration on some antioxidant parameters in the testis tissues of male New Zealand White Rabbits $(n=6)$

\begin{tabular}{|l|c|c|c|c|c|}
\hline & CONT & BPA & PUN & B $+P$ & $P$ \\
\hline MDA $(\mathrm{mmol} / \mathrm{L})$ & $1.69^{\mathrm{a}} \pm 0.70$ & $2.70^{\mathrm{b}} \pm 0.80$ & $1.14^{\mathrm{a}} \pm 0.45$ & $1.84^{\mathrm{ab}} \pm 8.93$ & 0.023 \\
SOD $(\mathrm{pg} / \mathrm{mL})$ & $363.0^{\mathrm{a}} \pm 58.2$ & $241.4^{\mathrm{b}} \pm 62.3$ & $391.6^{\mathrm{a}} \pm 73.9$ & $304.0^{\mathrm{ab}} \pm 74.1$ & 0.013 \\
\hline CAT $(\mathrm{pg} / \mathrm{mL})$ & $11.78^{\mathrm{ab}} \pm 1.09$ & $8.78^{\mathrm{c}} \pm 1.23$ & $13.7^{\mathrm{a}} \pm 4.83$ & $10.68^{\mathrm{bc}} \pm 2.85$ & 0.010 \\
GPX $(\mathrm{pg} / \mathrm{mL})$ & $2013^{\mathrm{ab}} \pm 276$ & $1543^{\mathrm{b}} \pm 276$ & $2209^{\mathrm{a}} \pm 551$ & $1638^{\mathrm{b}} \pm 245$ & 0.031 \\
\hline
\end{tabular}

Explanations: CONT - control; BPA - $20 \mathrm{mg} / \mathrm{kg} /$ day BPA; PUN - $2 \mathrm{mg} / \mathrm{kg} /$ day punicalagin; $\mathrm{B}+\mathrm{P}-20 \mathrm{mg} / \mathrm{kg} /$ day BPA and $2 \mathrm{mg} / \mathrm{kg} /$ day punicalagin; MDA - malondialdehyde; SOD - superoxide dismutase; CAT - catalase; GPx - glutathione peroxidase; a, b, c-rows with different letters differ significantly. Values are given as mean \pm standard deviation.

treated rabbits $(\mathrm{P}<0.03)$. However, estradiol, inhibin, $\mathrm{FSH} / \mathrm{LH}$, and $\mathrm{FSH} /$ inhibin ratios were not affected by any of the treatments $(\mathrm{P}>0.1)$. Similarly, there was no difference in ejaculate volume, ejaculate weight, and ejaculate $\mathrm{pH}$ among the groups. Nevertheless, the progressive motility and spermatozoa concentration was negatively altered by oral BPA. The decline in progressive motility and sperm concentration by BPA treatment was statistically increased to the control levels with punicalagin in the $\mathrm{B}+\mathrm{P}$ group. In addition, punicalagin treatment alone positively altered these two spermatological parameters over the control levels (Tab. 2).

There are different studies that attempt to reveal the relation between BPA and gonadotropins in humans and rodents. In a previous study, a positive correlation was found between high urinary BPA levels and serum FSH and testosterone levels in men, while a negative correlation with inhibin level was observed (19). In another study, BPA increased LH levels (17). On the other hand, BPA was reported to decrease serum LH levels in rats (32). Contrary to these previous studies, it is possible to find studies that do not encounter any relation between BPA and FSH and inhibin secretion (20). BPA had no effect on plasma LH and FSH levels in rats $(2,22,23)$. Similarly, in the current study, BPA did not show a significant effect on serum inhibin, FSH, and LH levels. Although changes in reproductive hormones in mammals can be perceived as a warning that there may be reproductive toxicity, it should be taken into consideration that even if these hormones are in their normal blood concentrations, there may be damage to the testicular tissue due to oxidative stress and this can lead to adverse effects on spermatogenesis $(16,23)$.

The oxidant-antioxidant enzymes measured in testis tissues are in Table 3. The levels of testicular MDA increased significantly in BPA treated groups when compared to the controls. Furthermore, when the control group and the BPA group were compared, the SOD, CAT, and GPx enzyme levels decreased significantly in the BPA groups. However, the negative alterations due to BPA treatments on testicular MDA, SOD, and CAT were prevented with punicalagin in the $\mathrm{B}+\mathrm{P}$ group. Intracellular enzymatic antioxidants such as SOD, CAT, and GPx have a key role in the removal of reactive oxygen species (26). Additionally, MDA is the most commonly used parameter to determine the degree of lipid peroxidation. Altered levels of MDA, SOD, and CAT were found in the testis tissues of BPA-treated rabbits compared to the control group. On the other hand, altered MDA, SOD, and CAT levels due to BPA in the testicular tissue return to the control levels with punicalagin treatment. This may typically be the result of increased lipid peroxidation as a result of oxidative stress due to BPA. BPA is known to suppress the enzymatic antioxidant defense system and increase lipid peroxidative dam- 
age in different organs $(5,14)$. In contrast, it has been demonstrated that punicalagin showed an important antioxidant activity in tissues exposed to oxidative stress (33). Thus, the results on testicular oxidant-antioxidant levels suggested that BPA could exhibit toxic effects by decreasing defense against oxidants, and punicalagin could reduce oxidative damage directly or indirectly by preventing increased free radical formation due to BPA.

The histopathological examination of the testis tissues revealed that BPA treatment alone caused testicular degeneration. There was a decrease in the spermatozoa number and an increase in the vacuolar degeneration due to BPA exposure. The degree of degeneration was less pronounced in the $\mathrm{B}+\mathrm{P}$ group: when coupled with BPA, punicalagin treatment tended to have positive effects on the histopathological alterations caused by BPA (Tab. 4). BPA showed similar alterations in the seminiferous tubules of the rats because of the increased oxidative stress $(12,15)$. Verma and Sangai (31) reported that BPA treatment increased the oxidative stress that leads to cell and membrane damage of human erythrocytes. Low doses of BPA induced oxidative stress in the liver tissues of rats (5). In the current study, testicular damage induced by BPA may be due to the ability of BPA to stimulate the generation of reactive oxygen species in the testis. The histopathological alterations due to BPA exposure could also be another factor affecting the negative changes in semen quality and substantiate the impaired spermatogenesis.

Sperm concentration and motility are vital parameters of male fertility. An optimal concentration and motility are needed for successful fertilization (29). Antioxidants hinder oxidation and thus prevent oxidative stress. There is ample evidence to support that BPA interferes with the reproductive health of humans and animals. In spermatozoa, BPA-induced adverse effects are most likely caused by increased oxidative stress because BPA could affect sperm quality by reducing antioxidant enzymes (7). It has been previously reported that antioxidants have protective roles in semen quality in different mammals (21). The positive effects of antioxidants on spermatological parameters also have been reported within in vitro models $(8,24$, 27). Similarly to previous findings, the results from the current study suggested that the decrease in sperm concentration and motility due to BPA exposure was mostly recovered by a powerful antioxidant-punicalagin. Furthermore, elevated testicular oxidative stress in BPA-treated rabbits was recovered by punicalagin. An increase in testicular oxidative stress could cause an injury in sperm cell integrity and reduce the number and/or motility of these cells $(1,25)$. Thus, BPA can stimulate free radical formation in Male New Zealand rabbits and punicalagin could prevent BPA-induced excessive oxidative stress in the testis tissues, and consequently maintaining sperm integrity and motility.

In conclusion, the present study suggests that punicalagin has a positive effect against oxidative stress
Tab. 4. Histopathological changes in testicular tissues due to bisphenol A (BPA) and/or punicalagin (PUN) treatments $(n=6)$

\begin{tabular}{|l|c|c|c|c|}
\hline & CONT & BPA & PUN & B+P \\
\hline $\begin{array}{l}\text { Decrease in the spermatozoa } \\
\text { number in the tubulus } \\
\text { seminiferus contortus lumen }\end{array}$ & $\begin{array}{r}-(5 / 6) \\
+(1 / 6)\end{array}$ & $\begin{array}{r}++(3 / 6) \\
+++(3 / 6)\end{array}$ & $-(6 / 6)$ & $\begin{array}{r}-(3 / 6) \\
+(3 / 6)\end{array}$ \\
$\begin{array}{l}\text { Increase in the vacuolar } \\
\text { degeneration }\end{array}$ & $\begin{array}{r}-(5 / 6) \\
+(1 / 6)\end{array}$ & $\begin{array}{r}+(1 / 6) \\
++(2 / 6) \\
+++(3 / 6)\end{array}$ & $-(6 / 6)$ & $\begin{array}{r}-(2 / 6) \\
+(4 / 6)\end{array}$ \\
\hline
\end{tabular}

Explanations: CONT - control; BPA - $20 \mathrm{mg} / \mathrm{kg} /$ day BPA; $\mathrm{PUN}-2 \mathrm{mg} / \mathrm{kg} /$ day punicalagin; $\mathrm{B}+\mathrm{P}-20 \mathrm{mg} / \mathrm{kg} /$ day BPA and $2 \mathrm{mg} / \mathrm{kg}$ /day punicalagin; - absent or minimal; + mild; ++ moderate; +++ marked

stimulated by BPA in testicular tissue of rabbits. As a result, punicalagin has a protective effect on testicular tissue against BPA's oxidative stress. The protective effect of punicalagin may be due to an increase in the activity of the antioxidant defense system and/ or inhibition of lipid peroxidation. This suggests that punicalagin may be effective in preventing possible negative effects of BPA on the reproductive system.

\section{References}

1. Agarwal A., Virk G., Ong C., du Plessis S. S.: Effect of oxidative stress on male reproduction. World J. Mens Health 2014, 32, 1-17.

2. Akingbemi B. T., Sottas C. M., Koulova A. I., Klinefelter G. R., Hardy M. P.: Inhibition of testicular steroidogenesis by the xenoestrogen bisphenol A is associated with reduced pituitary luteinizing hormone secretion and decreased steroidogenic enzyme gene expression in rat Leydig cells. Endocrinology 2004, $145,592-603$

3. Ata A., Hatipoglu F. S., Yildiz-Gulay O., Gulay M. S.: Protective role of ascorbic acid on subacute sperm toxicity in male New Zealand white rabbits treated with endosulfan. Drug Chem. Toxicol. 2007, 30, 181-195.

4. Ata A., Yildiz-Gulay O., Gungor S., Balic A., Gulay M. S.: The effects of carob (Ceratonia Siliqua) bean extract on male New Zealand White rabbit semen. World Rabbit Sci. 2018, 26, 209-215.

5. Bindhumol V., Chitra K. C., Mathur P. P.: Bisphenol a induces reactive oxygen species generation in the liver of male rats. Toxicology 2003, 188, 117-124.

6. Chen D., Kannan K., Tan H., Zheng Z., Feng Y. L., Wu Y., Widelka M.: Bisphenol Analogues Other Than BPA: Environmental Occurrence, Human Exposure, and Toxicity - A Review. Environ. Sci.Technol. 2016, 50, 5438-5453.

7. Chitra K. C., Latchoumycandane C., Mathur P. P.: Induction of oxidative stress by bisphenol A in the epididymal sperm of rats. Toxicology 2003, 185, 19-127.

8.Fanaei H., Khayat S., Halvaei I., Ramezani V., Azizi Y., Kasaeian A., Mardaneh J., Parvizi M. R., Akrami M.: Effects of ascorbic acid on sperm motility, viability, acrosome reaction and DNA integrity in teratozoospermic samples. Iran J. Reprod. Med. 2014, 12, 103-110.

9. Geens T., Aerts D., Berthot C., Bourguignon J. P., Goeyens L., Lecomte P., Covaci A.: A review of dietary and non-dietary exposure to bisphenol-A. Food Chem. Toxicol. 2012, 50, 3725-3740.

10. Gil M. I., Tomás-Barberán F. A., Hess-Pierce B., Holcroft D. M., Kader A. A.: Antioxidant activity of pomegranate juice and its relationship with phenolic composition and processing. J. Agric. Food Chem. 2000, 48, 4581-4589.

11. Gonçalves G. D., Semprebon S. C., Biazi B. I., Mantovani M. S., Fernandes G. S. A.: Bisphenol A reduces testosterone production in TM3 Leydig cells independently of its effects on cell death and mitochondrial membrane potential. Reprod. Toxicol. 2018, 76, 26-34.

12. Hassan A. H., Ismail A. A., Khudir A. N.: Effects of pre-and postnatal exposure to Bisphenol-A on the reproductive efficacy in male albino rats. J. Kerbala Univers. 2013, 11, 1-15.

13.Jahan S., Ain Q. U., Ullah $H$.: Therapeutic effects of quercetin against bisphenol A induced testicular damage in male Sprague Dawley rats. Syst. Biol. Reprod. Med. 2016, 62, 14-124.

14. Kabuto H., Amakawa M., Shishibori T.: Exposure to bisphenol A during embryonic/fetal life and infancy increases oxidative injury and causes underdevelopment of the brain and testis in mice. Life Sciences 2004, 74, 2931-2940. 
15. Kamel A. H., Foaud M. A., Moussa H. M. T.: The adverse effects of bisphenol A on male albino rats. J. Basic and Applied Zoology 2018, 79, 6, https://doi. org/10.1186/s41936-018-0015-9.

16. Karabulut H., Gulay M. S.: Influence of bisphenol A on spermatalogical parameters of New Zealand White Rabbits. Med. Weter. 2020, 76, 326-330.

17. Lassen T. H., Frederiksen H., Jensen T. K., Petersen J. H., Joensen U. N., Main K. M.: Urinary bisphenol A levels in young men: Association with reproductive hormones and semen quality. Environ Health Perspect. 2014, 122, 478-484.

18. Li D. K., Zhou Z., Miao M., He Y., Qing D., Wu T., Wang J., Weng X., Ferber J., Herrinton L. J., Zhu Q., Gao E., Yuan W.: Relationship Between Urine Bisphenol-A Level and Declining Male Sexual Function. J. Androl. 2010, 31 , 500-506.

19. Meeker J. D.: Exposure to environmental endocrine disrupting compounds and men's health. Maturitas 2010, 66, 236-241.

20. Mendiola J., Jørgensen N., Andersson A. M., Calafat A. M., Ye X., Redmon J. B., Drobnis E. Z., Wang C., Sparks A., Thurston S. W., Swan S. H.: Are Environmental Levels of Bisphenol AAssociated with Reproductive Function in Fertile Men? Environ. Health Perspect. 2010, 118, 1286-1291.

21. Mortazavi M., Salehi I., Alizadeh Z., Vahabian M., Roushandeh A. M. Protective Effects of Antioxidants on Sperm Parameters and Seminiferous Tubules Epithelium in High Fat-fed Rats. J. Reprod. Infertil. 2014, 15, 22-28.

22. Nakamura D., Yanagiba Y., Duana Z., Itoa Y., Okamura A., Asaeda N. Tagawa Y., Lic C. M., Taya K., Zhanga S. Y., Naitoa H., Ramghana D. H., Kamijima M., Nakajima T.: Bisphenol A may cause testosterone reduction by adversely affecting both testis and pituitary systems similar to estradiol. Toxicol. Lett. 2010, 194, 16-25.

23. Ogo F. M., Siervo G. E. M. L., Gonçalves G. D., Cecchini R., Guarnier F. A., Anselmo-Franci J. A., Fernandes G. S. A.: Low doses of bisphenol A can impair postnatal testicular development directly, without affecting hormonal or oxidative stress levels. Reprod. Fertil. Dev. 2017, 29, 2245-2254.

24. Rochester J. R.: Bisphenol A and human health: a review of the literature. Reprod. Toxicol. 2013, 42, 132-155.

25. Sabeti P., Pourmasumi S., Rahiminia T., Akyash F., Talebi A. R.: Etiologies of sperm oxidative stress. Int. J. Reprod. Biomed. 2016, 14, 231-240.
26. Sen S., Chakraborty R., Sridhar C., Reddy Y. S. R., De B.: Free radicals, antioxidants, diseases and phytomedicines: Current status and future prospect. Int. J. Pharm. Sci. Res. 2010, 3, 91-100.

27. Shah N., Singh V., Yadav H. P., Verma M., Chauhan D. S., Saxena A., Yadav S. Swain D. K.: Effect of reduced glutathione supplementation in semen extender on tyrosine phosphorylation and apoptosis like changes in frozen thawed Hariana bull spermatozoa. Anim. Reprod. Sci. 2017, 182, 111-122.

28. Sharpe R. M.: Environmental/lifestyle effects on spermatogenesis. Philos. Trans. R. Soc. B. Biol. Sci. 2010, 365,1697-1712.

29. Suarez S. S.: Control of hyperactivation in sperm. Hum. Reprod. Update 2008 14, 647-657.

30. Verma A., Kanwar K. C.: Effect of vitamin E on human sperm motility and lipid peroxidation in vitro. Asian J. Androl. 1999, 1, 151-154.

31. Verma R. J., Sangai N. P.: The ameliorative effect of black tea extract and quercetin on bisphenol A-induced cytotoxicity. Acta Poloniae Pharmaceutica 2009, 66, 41-44.

32. Wisniewski P., Romano R. M., Kizys M. M. L., Oliveira K. C., Kasamatsu T. Giannocco G., Chiamolera M. I., Dias-Da-Silva M. R., Romano M. A.: Adult exposure to bisphenol A (BPA) in Wistar rats reduces sperm quality with disruption of the hypothalamic-pituitary-testicular axis. Toxicology 2015 , 329, 1-9.

33. Yaidikar L., Byna B., Thakur S. R.: Neuroprotective Effect of Punicalagin against Cerebral Ischemia Reperfusion-induced Oxidative Brain Injury in Rats. J. Stroke Cerebrovas. Diseases 2014, 23, 2869-2878.

34. Yildiz-Gulay O., Gulay M. S.: Punicalagin is beneficial for spermatogenesis in male New Zealand White rabbits. Indian J. Anim. Res. 2019, 53, 457-460

35. Yin L., Dai Y., Cui Z., Jiang X., Liu W., Han F., Lin A., Cao J., Liu J.: The regulation of cellular apoptosis by the ROS-triggered PERK/EIF $2 \alpha /$ chop pathway plays a vital role in bisphenol A-induced male reproductive toxicity. Toxicol. Appl. Pharmacol. 2017, 314, 98-108

Corresponding author: Prof. Dr. Mehmet Sukru GULAY; Burdur Mehmet Akif Ersoy University, Faculty of Veterinary Medicine, Department of Physiology, Istiklal Yerleskesi, Burdur/Turkey; e-mail: msgulay@ mehmetakif.edu.tr 